\title{
Activator protein 1-mediated transcriptional regulation strategy sustains long-term expression of a xenogeneic gene product in vivo: An implication for gene therapy targeting congenital protein deficiencies
}

\author{
SHIN SASAKI ${ }^{1}$, JAMES M. SMITH $^{2}$, KEIKO TAKASE $^{1}$, KENJI OKUDA $^{1}$, \\ NORIHISA ISHII ${ }^{3}$ and FUMIHIKO TAKESHITA ${ }^{1}$
}

\author{
${ }^{1}$ Department of Molecular Biodefense Research, Yokohama City University Graduate School of Medicine, \\ Yokohama 236-0004, Japan; ${ }^{2}$ Antibody Systems, Inc. Hurst, Texas 76054, USA; ${ }^{3}$ Department of Bioregulation, \\ Leprosy Research Center, National Institute of Infectious Diseases, Tokyo 189-0002, Japan
}

Received March 7, 2006; Accepted April 20, 2006

\begin{abstract}
Maintenance of high-level transgene expression is the main challenge in current gene therapy. Although the cytomegalovirus (CMV) promoter/enhancer or its derivative the CAG promoter has been harnessed in current gene therapy vectors, transgene expression by these vectors is often transient and remains at suboptimal levels due to undefined mechanisms, possibly including the shortage of transcriptional machinery. To overcome this drawback, we designed a novel transcriptional control system, designated here as transcription factorsupercharging promoter system, in which transgene expression is regulated by the positive feedback circuit consisting of cisand trans-acting elements of gene expression machinery. Among combinations of these elements, a plasmid composed of a target gene expression cassette driven by the chimeric CMV promoter containing repetitive 12-O-tetradecanoylphorbol-13acetate-responsive elements as cis-acting elements (CMV-TTT) and expression cassettes for c-Fos and c-Jun genes as transacting elements facilitated high and long-term ( $>10$ months) expression of a transgene after its intramuscular electroporation-mediated delivery in mice. Since human secretory alkaline phosphatase was used as a reporter, it was suggested that the immune evasion mechanism elicited by the CMV-TTT and/or c-Fos/ c-Jun expression also contributed to the sustained expression in mice. Our strategy may open a new avenue for a gene therapy that involves lifelong supplementation of a deficient protein that could be targeted by the host's immune system.
\end{abstract}

Correspondence to: Dr Fumihiko Takeshita, Department of Molecular Biodefense Research, Yokohama City University Graduate School of Medicine, Yokohama 236-0004, Japan

E-mail: takesita@yokohama-cu.ac.jp

Key words: promoter, AP-1, gene therapy

\section{Introduction}

Achievement of sustainable transgene expression in vivo by non-viral transfer approach is a major goal in current gene therapy research. The magnitude of transgene expression mainly depends upon the promoter activity, thus strong promoters such as CMV (1) or CAG (2) have been preferentially used in the study pursuing in vivo transgene expression. In fact, these promoters attain strong expression of a transgene shortly after DNA delivery, but this expression rapidly dissipates within several weeks (3-8). A potential mechanism behind this downregulation is immune-mediated elimination of the cells bearing exogenous gene products $(5,7-9)$.

This may raise a significant concern when considering gene therapy for humans, since proteins responsible for congenital deficiencies are often recognized as foreign by the patient's immune system. It is reported that enzyme replacement therapy induces an immune response to deficient proteins in patients with a wide range of congenital deficiencies such as hemophilia A and B (10), adenosine deaminase deficiency (11), mucopolysaccharidosis type I (12), Gaucher disease (13), Fabry disease (14), and Pompe disease (15). Hence, regulation of the host immune system is a key for successful gene therapy targeting congenital protein deficiencies. An attractive approach avoiding elicitation of the immune response against a gene product is the use of a target tissue-specific promoter that is generally inert in antigen presenting cells. A number of tissue-specific promoters have been tested in various models $(16,17)$, however, these promoters are too weak to induce target gene expression at a therapeutic level. Accordingly, it is of importance to establish a strategy that can induce robust transgene expression but not elicit immune response against a transgene product. In the present study, we sought to establish a novel transcriptional control system that attained potent and sustainable exogenous gene expression in vivo.

Tandem repeats of an NF- $\mathrm{NB}$ or AP-1 binding element were introduced into the $\mathrm{CMV}$ promoter which drives the target gene, and then $\mathrm{NF}_{-} \mathrm{KB}$ or AP-1 was provided by $\mathrm{cis}$ existing expression cassette. This system, designated as the 
transcription factor (TF)-supercharging promoter system, allowed creation of a transcriptional feedback circuit by cisacting elements within the promoter and corresponding transacting factors expressed exogenously, which drives mandatory expression of a transgene. Our results demonstrated that the AP-1-mediated supercharging promoter system can attain enhanced and prolonged xenogeneic human SEAP expression in mice while the conventional vector failed to sustain the expression. This system may be well suited for applications where high-level and long-lasting therapeutic gene expression is required, especially in patients who may develop an immune response to a therapeutic protein during enzyme replacement therapy.

\section{Materials and methods}

Plasmid vectors. The parental vector used in plasmid construction is pGA (18) that consists of CMV promoter plus intron $\mathrm{A}$, bovine growth hormon terminator, Kanr, and pUC backbone. Fig. 2 shows a schematic illustration of the supercharging promoter characterized in the current study. Unique restriction sites were created in the CMV promoter using a QuickChange site-directed mutagenesis kit (Stratagene, La Jolla, CA) to allow insertion of a transcriptional activator binding element (Fig. 2A). Tandem repeats of transcription factor (TF) binding motif, five tandem repeats of the $\kappa \mathrm{B}$ element ( $\mathrm{K}$ motif) or seven tandem repeats of the 12-Otetradecanolyphorbol 13 acetate-responsive element (T motif) were from PathDetect in vivo cis-reporting system (Stratagene). Each motif was amplified by PCR and digested with the corresponding restriction enzymes, and then ligated into an appropriate region of the CMV promoter as illustrated in Fig. 2B and 2C. Reporter genes, firefly luciferase (luc) and human secretory alkaline phosphatase (huSEAP) were subcloned from pGL3 control (Promega, Madison, WI) and pSEAP2-control (BD Clontech, Palo Alto, CA), respectively. LacZ and chloramphenicol acetyl transferase (CAT) used as a mock insert were obtained from $\mathrm{pSV}$-ß-galactosidase and pCAT3-basic (both from Promega), respectively. Mouse SEAP (muSEAP) was cloned from $\mathrm{C} 2 \mathrm{C} 12$ murine myoblast as reported by Wang et al (19). Mouse RelA, cFos, and cJun were cloned from mouse spleen cDNA library as described elsewhere (20). Dual or triple promoter vectors were constructed by ligation of two or three expression cassettes in tandem as described previously (21).

Transfection and luciferase assay. The cell lines used in this study are listed in Table I. Each cell line (cultured in DMEM high-glucose supplemented with $10 \%$ FCS) was seeded in a 96-well tissue culture plate and grown until reaching 50-70\% confluency. Then cells were transiently transfected using FuGene 6 (Roche, Mannheim, Germany) in triplicate. Each transfection mixture contained different dose combinations of reporter, transcription activator, and mock plasmid. Two days following transfection, the luminescence signal was measured by a microplate luminescence reader (Fluostar, BMG Labtechnologies, Duren, Germany) with a Bright-Glo kit (Promega) according to the manufacturer's instruction. A secondary reporter (ex. Renilla luciferase) for standardization of primary reporter activity was not employed in this work,
Table I. Cell lines used for transient transfection experiments.

\begin{tabular}{lll}
\hline Cell lines & Species & Tissue \\
\hline HEK293 & Human & Embryonic kidney \\
HeLa & & Uterine cervical carcinoma \\
HepG2 & & Hepatoma \\
A431 & & Epidermoid carcinoma \\
NIH-3T3 & Mouse & Embryonic fibroblast \\
C2C12 & & Myoblast \\
ST2 & & Bone marrow stroma \\
C6 & Rat & Glioma \\
CHO-K1 & Chinese hamster & Ovary \\
\hline
\end{tabular}

since we found that commonly used promoters (ex. CMV, CAG, SV40, and HSV-TK) were susceptible to cotransfected TF plasmid. Therefore any secondary reporters seemed not to serve as an internal control, and we intended to eliminate experimental variations by repeating experiments.

Animal treatment and measurement of plasma SEAP activity. Male BALB/c and SCID mice were purchased from Japan Clea and housed in the animal facility of the Leprosy Research Center, National Institute of Infectious Diseases or Yokohama City University Graduate School of Medicine. DNA injection, electroporation, and retro-orbital puncture for blood sampling were all performed under anesthesia by subcutaneous injection of the 4:1 mixture of Ketamine and Xylazine. Animal experiments were conducted under the approval of the institutional laboratory animal care and use committee. In electroporation, the quadriceps femoris muscle was exposed and injected with the indicated dose of plasmid in saline with a 30 -gauge needle inserted 2-3 $\mathrm{mm}$ deep, then the entire muscle bundle was pinched by a pair of disk-type platinum electrodes (diameter: $10 \mathrm{~mm}$, separation: 5 mm, CUY650-10; Nepa Gene, Chiba, Japan). Six electrical pulses were applied at $30 \mathrm{~V}$ by a square wave electroporator CUY21EDIT (Nepa Gene), with pulse duration of $50 \mathrm{~ms}$, pulse interval of $100 \mathrm{~ms}$, and reversal of polarity after three pulses, followed by the closure of incision with surgical wound clips. Scheduled blood samples were taken by retro-orbital punctuation and stored at $-20^{\circ} \mathrm{C}$ until assayed. Plasma SEAP activity was measured using an SEAP reporter gene chemiluminescent assay kit (Roche) using a microplate luminescence reader according to the manufacturer's instruction.

$R N A$ extraction and real-time PCR. To explore the transgene expression pattern in muscle and lymphoid tissue by different transcriptional control, transgene expression was quantified by real-time RT-PCR at the messenger RNA level. To eliminate immune-mediated loss of transgene expression, mouse SEAP was used in this experiment. One or three days following electroporation-mediated DNA delivery, mice were sacrificed and the inguinal lymph nodes and muscles were harvested. RNA was extracted with TRIzol reagent (Invitrogen, Life 


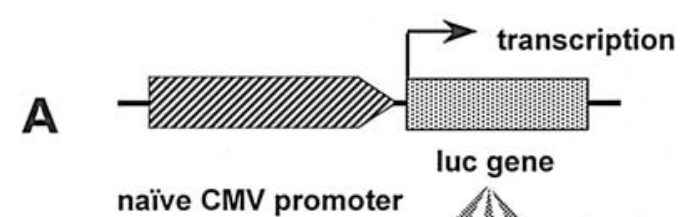

naîve CMV promoter

\& luminescence signal

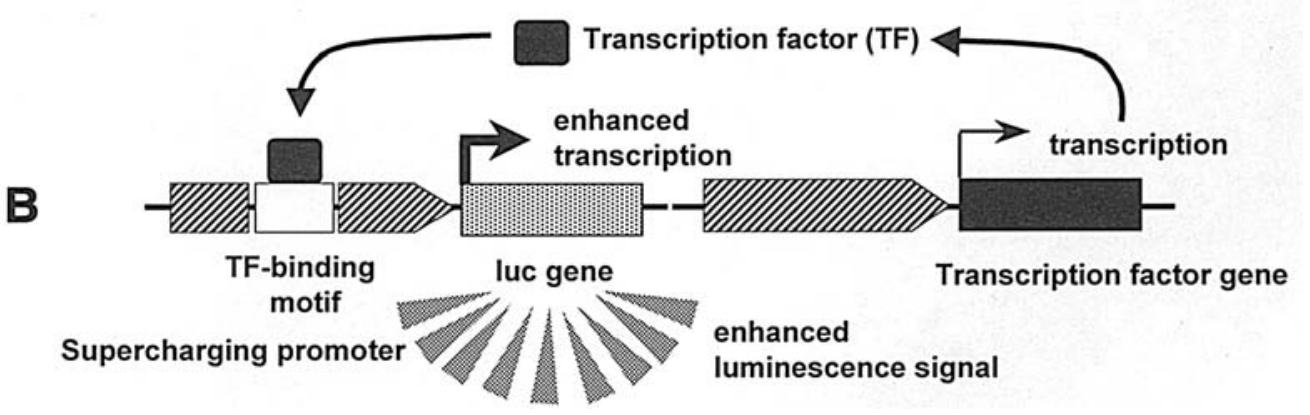

Figure 1. Schematic diagram of the transcription factor (TF)-supercharging promoter system. (A) A conventional transgene expression cassette. The firefly luciferase (luc) gene is expressed from the cytomegalovirus enhancer/promoter, which can be quantified by the luminescence signal. (B) TF-supercharging promoter system. Two expression cassettes need to be delivered into the target cell. One expresses a TF, which binds onto a TF-binding site(s) artificially introduced into the CMV promoter, and this results in trans-activation and the enhancement of luciferase gene expression.

Technologies, Carlsbad, CA) in accordance with the manufacturer's instructions. Before cDNA synthesis, each RNA sample was treated with DNase I (Invitrogen Life Technologies) to eliminate any potential contamination with plasmid or genomic DNA. Reverse transcription was performed with $2 \mu \mathrm{g}$ of total RNA using a superscript II reverse transcription amplification system (Life technologies, Invitrogen) with random hexamers. Real-time PCR amplification was performed on an ABI PRISM 7700 using SYBRGreen dye. PCR conditions were $50^{\circ} \mathrm{C}$ for $2 \mathrm{~min}, 95^{\circ} \mathrm{C}$ for $10 \mathrm{~min}$, then 40 cycles at $95^{\circ} \mathrm{C}$ for $15 \mathrm{sec}$, and $60^{\circ} \mathrm{C}$ for $1 \mathrm{~min}$. The primer set 5'-ATG TGG GGA GCC TGC TTG CTG-3' (forward) and 5'-CCA GAT GGC CTT GCT GCT GCC-3' (reverse) was used to amplify a 244-bp fragment of the mouse SEAP mRNA gene. For quantitative evaluation of gene expression, mouse SEAP mRNA levels were normalized to the 18S-mRNA as suggested by Applied Biosystems.

Statistics. Statistical analysis was conducted with one-way analysis of variance (ANOVA) or paired t-test using StatView J-4.02 software (Abacus Concepts, Berkeley, CA), and significance was defined when $\mathrm{p}<0.05$.

\section{Results}

Strategy for transcriptional control. The principle for the supercharging promoter system is shown in Fig. 1. In the conventional expression system, a single promoter drives the transcription of a target gene (Fig. 1A). To ameliorate the promoter activity of current gene therapy vectors, we sought to establish a novel system that would allow mandatory expression of a transgene as shown in Fig. 1B. A transcription factor binding motif (enhancer site) was introduced into the CMV promoter, then a corresponding transcription factor was supplied by cis-existing expression cassette. This was expected to induce robust transgene expression by the action of positive-feedback circuit composed of cis- (enhancer sites) and trans- (TFs) acting elements. To test the principle of this strategy, the promoters CMV-K, CMV-KK, CMV-KKK, CMV-T, CMV-TT, and CMV-TTT were constructed by introducing $\mathrm{K}$ or $\mathrm{T}$ motif into their $\mathrm{CMV}$ promoter regions as illustrated in Fig. $2 \mathrm{~B}$ and $\mathrm{C}$. NF- $\mathrm{\kappa B}$ is a pivotal transcriptional regulator in controlling innate and adaptive immunity (22) while AP-1 regulates genes involved in cell growth (23). Among several TF candidates for each TF-binding site, we used RelA (p65) for K motif and c-Fos and c-Jun heterodimer for $\mathrm{T}$ motif, since these combinations demonstrated a potent trans-activity in preliminary in vitro experiments (data not shown).

Transgene expression by the supercharging promoter system in vitro. To determine if this approach is capable of ameliorating transgene expression performance, HEK293 and NIH$3 \mathrm{~T} 3$ cells were transiently cotransfected with a reporter and corresponding TF expression plasmid(s) (equimolar cFos and cJun plasmids were applied for AP-1). As shown in Fig. 3A, luc expression in HEK 293 cells increased in accordance with increasing the number of $\mathrm{K}$ motifs in a promoter, whereas these promoters showed a marginal effect in NIH-3T3 cells. In contrast, introduction of $\mathrm{T}$ motifs successfully augmented luc expression in both cell lines (Fig. 3B). These results suggest that the effect of co-expressed TF may depend on cell type. We also sought to determine how many copies of $\mathrm{K}$ or $\mathrm{T}$ motifs were optimal for the postive-feedback effect. No statistical differences were noted in the expression level between CMV-KKK and -KK or between CMV-TTT and -TT. Simultaneously, significant differences were found between CMV-KK and -K in HEK 293 cells and CMV-TT and -T in both cells, indicating that two copies of $\mathrm{K}$ or $\mathrm{T}$ motifs might be sufficient. To verify if this observation is consistent across cell lines, the same experiments were conducted using $\mathrm{C} 2 \mathrm{C} 12$, C6, HeLa, HepG2, and ST2 cells, which gave similar results 


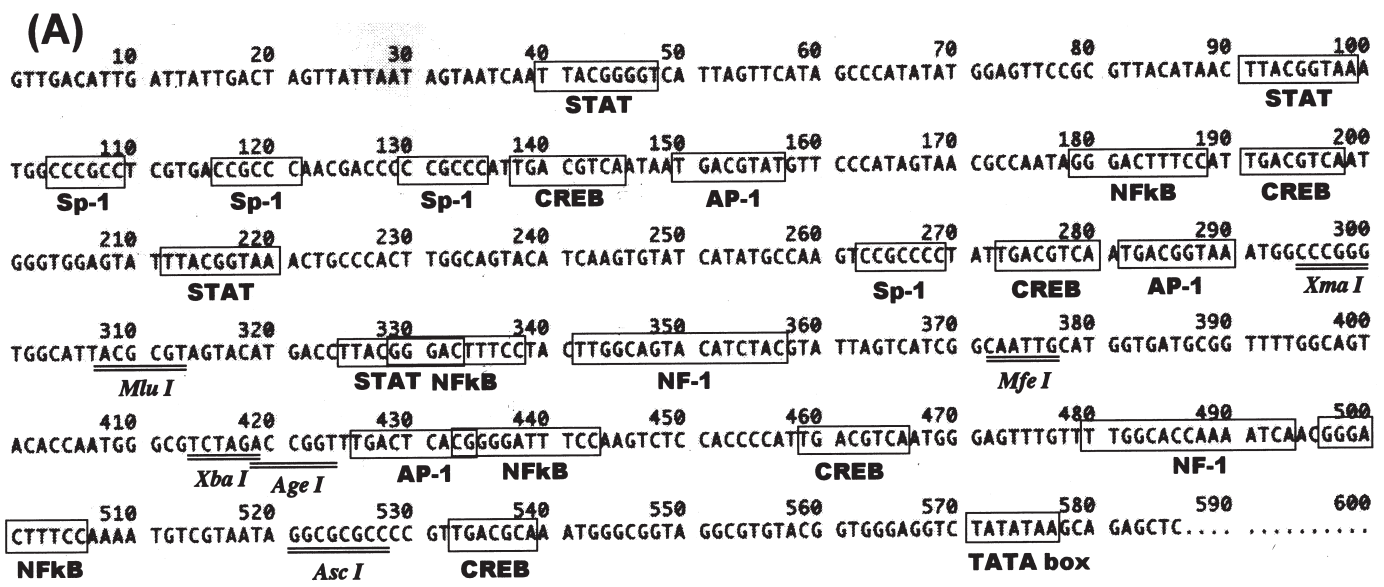

(B)
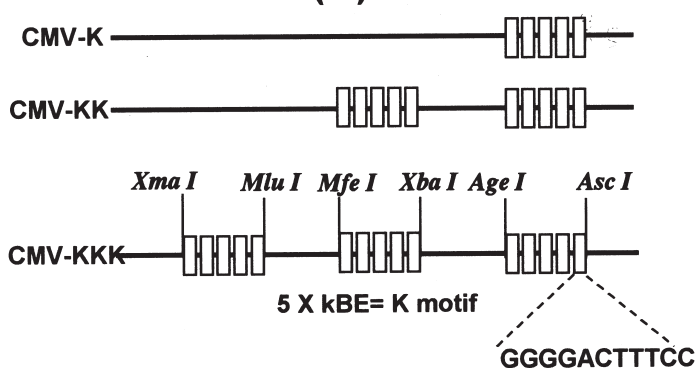

(C)

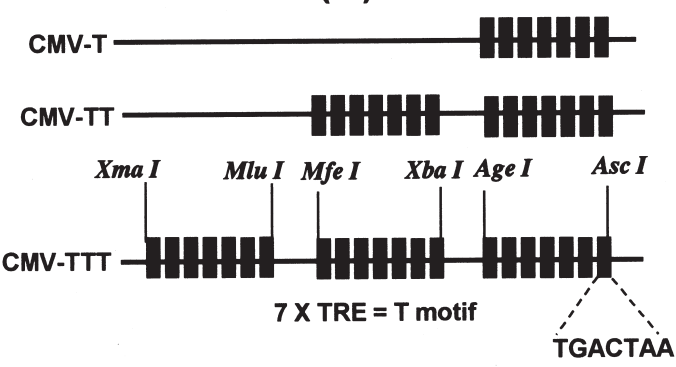

Figure 2. (A) Sequence of the human cytomegalovirus immediate-early gene enhancer/promoter that has additionally created restriction sites (double underlined). Original existing transcription factor binding sites are boxed. (B) Structure of the CMV promoter that has expanded NF- $\mathrm{B}$ binding elements (GGGACTTTCC; kBE). Five kBE tandem repeats (K motifs) are introduced between AgeI and AscI, MfeI and XbaI, and XmaI and MluI sites to create the CMV-KKK promoter. As shown, the CMV-K and CMV-KK promoters have one and two K motif(s), respectively. (C) Structure of the CMV promoter that has expanded AP-1 binding elements (TGACTAA; TRE). Seven TRE tandem repeats (T motifs) are introduced between AgeI and AscI, MfeI and XbaI, and XmaI and MluI sites to create the CMV-TTT promoter. As shown, the CMV-T and CMV-TT promoters have one and two T motif(s), respectively.

Table II. Evaluation of an NF-кB and AP-1-mediated supercharging promoter system in various cell lines.

\begin{tabular}{|c|c|c|c|c|c|c|c|c|}
\hline \multicolumn{2}{|c|}{ Transfected DNA } & \multicolumn{7}{|c|}{ Cell lines } \\
\hline $\begin{array}{l}\text { Reporter plasmid } \\
\text { (5 ng/well) }\end{array}$ & $\begin{array}{l}\text { Transcription activator } \\
\text { plasmid (50 ng/well) }\end{array}$ & $\mathrm{C} 2 \mathrm{C} 12$ & C6 & A431 & $\mathrm{HeLa}$ & HepG2 & ST2 & CHO-K1 \\
\hline \multirow[t]{3}{*}{ CMV/luc } & $\mathrm{CMV} / \mathrm{LacZ}$ & 1.0 & 1.0 & 1.0 & 1.0 & 1.0 & 1.0 & 1.0 \\
\hline & $\mathrm{CMV} / \mathrm{Rel} \mathrm{A}$ & $1.8 \pm 0.2$ & $6.5 \pm 0.5$ & $3.7 \pm 0.6$ & $7.2 \pm 0.9$ & $10.2 \pm 1.9$ & $2.1 \pm 0.1$ & $1.6 \pm 0.1$ \\
\hline & CMV/c-Fos + c-Jun & $21.4 \pm 3.1^{\mathrm{a}}$ & $2.1 \pm 0.3$ & $1.6 \pm 0.3$ & $1.8 \pm 0.3$ & $3.3 \pm 0.7$ & $5.9 \pm 1.1$ & $1.1 \pm 0.1$ \\
\hline \multirow[t]{2}{*}{ CMV-KKK/luc } & $\mathrm{CMV} / \mathrm{LacZ}$ & $0.4 \pm 0.1$ & $1.7 \pm 0.3$ & $2.9 \pm 0.5$ & $0.6 \pm 0.1$ & $4.5 \pm 1.4$ & $0.4 \pm 0.1$ & $<0.1$ \\
\hline & $\mathrm{CMV} / \mathrm{Rel} \mathrm{A}$ & $6.9 \pm 1.1^{\mathrm{a}}$ & $24.6 \pm 3.0^{\mathrm{a}}$ & $9.5 \pm 1.9$ & $13.7 \pm 1.5^{\mathrm{a}}$ & $16.0 \pm 3.3$ & $7.0 \pm 0.5^{\mathrm{a}}$ & $1.3 \pm 0.2$ \\
\hline \multirow[t]{2}{*}{ CMV-TTT/luc } & $\mathrm{CMV} / \mathrm{LacZ}$ & $2.2 \pm 0.2$ & 0.3 & $<0.1$ & $2.5 \pm 0.5$ & $1.8 \pm 0.4$ & $0.7 \pm 0.2$ & $<0.1$ \\
\hline & CMV/c-Fos + c-Jun & $27.5 \pm 3.4^{\mathrm{a}}$ & $7.1 \pm 1.0^{\mathrm{a}}$ & $0.8 \pm 0.1$ & $4.6 \pm 0.8$ & $7.4 \pm 1.7$ & $22.1 \pm 3.2^{\mathrm{a}}$ & $0.2 \pm 0.1$ \\
\hline
\end{tabular}

The cells were transiently transfected with the indicated plasmids, and firefly luciferase activities were measured 2 days following transfection. Values were standardized individually for each cell line by using the negative control group (CMV/luc + CMV/LacZ) to provide a basis for comparison among groups. The experiments were repeated at least three times with similar results. The data shown are

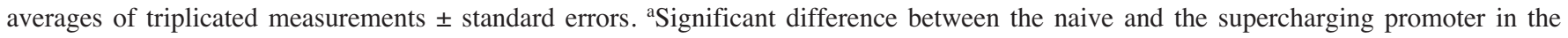
presence of an appropriate transcription activator plasmid.

(data not shown). These results suggest that two or three copies of $\mathrm{K}$ or $\mathrm{T}$ motifs are optimal for the system. Accordingly, CMV-KKK and CMV-TTT promoters were used in further experiments to determine the dose-response relationship between the amount of cotransfected TF plasmid and the activity of the chimeric promoter. 

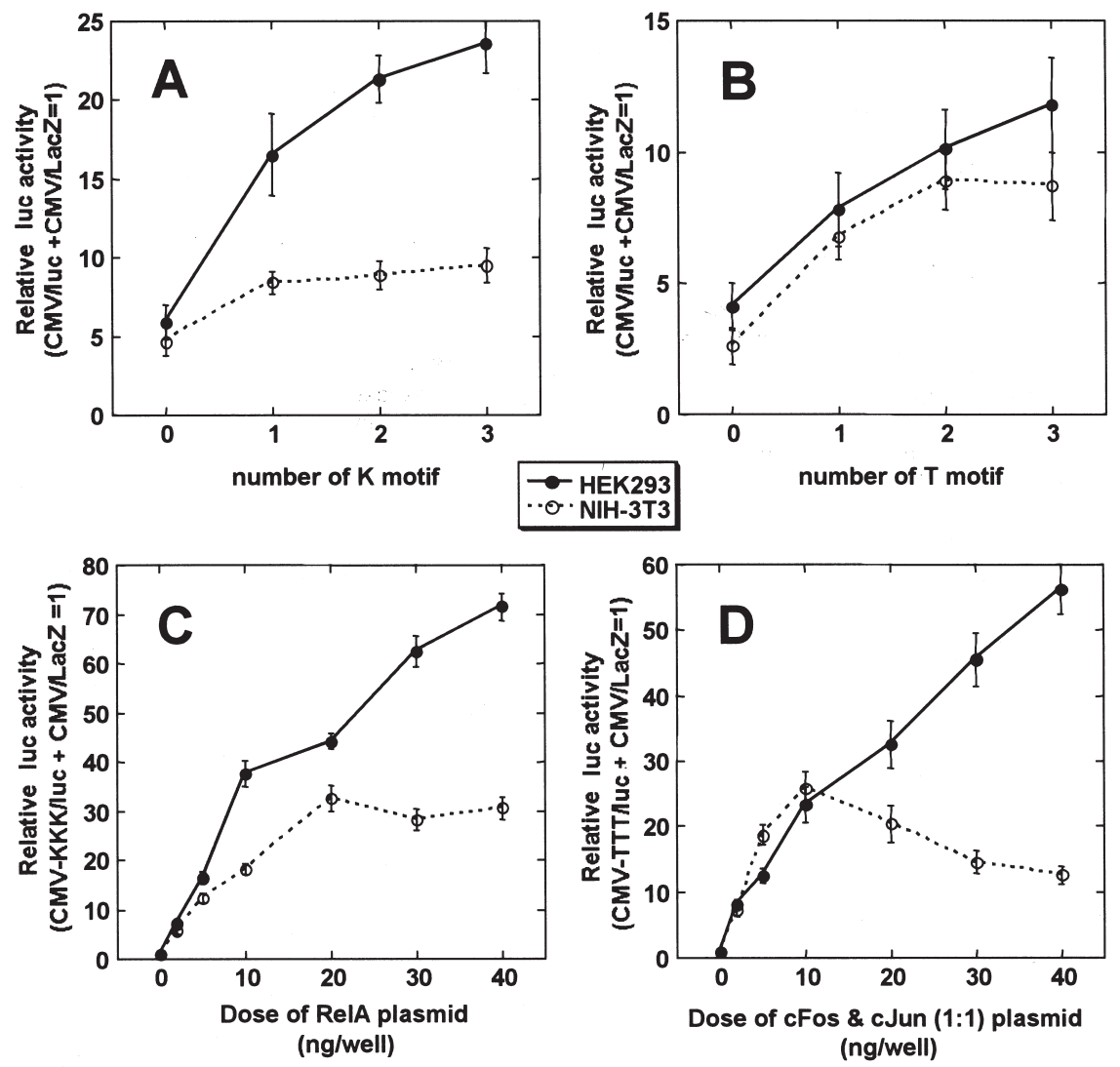

Figure 3. The supercharging promoter system accelerates the transgene expression in HEK293 and NIH-3T3 cells. CMV promoters containing one to three copies of the $\mathrm{K}$ motif (A) or $\mathrm{T}$ motif (B) were examined for their ability to enhance luc expression in the presence of the corresponding transcriptional activator plasmid (Rel A for the K motif, AP-1 for the T motif). The cells were cotransfected with a reporter (10 ng/well) and a transcription activator plasmid(s) (50 ng/well, for AP-1, equimolar amount of c-Fos and c-Jun plasmid), and luc activity was measured 2 days following transfection. The transcription factor dose-dependent luc expression kinetics controlled by the CMV-KKK (C) and CMV-TTT (D) promoters were also analysed 2 days following transfection. The dose of the transcription activator plasmid was increased from 0 to $40 \mathrm{ng} / \mathrm{well}$, while the dose of the reporter plasmid was maintained at $1 \mathrm{ng} /$ well. To make the transfected DNA amount constant, 40 to $0 \mathrm{ng} / \mathrm{well}$ of the mock plasmid (CMV/LacZ) was also contransfected. All values were standardized individually for each cell line using an appropriate negative control to provide a basis for comparison. The data shown are averages of triplicated measurements, and error bars indicate the standard deviations. The experiments were repeated twice with similar results.

Fig. 3C shows luc expression by the CMV-KKK promoter in the presence of various doses of RelA plasmid. In HEK 293 cells, a linear increase of luc activity was found with increasing doses of RelA plasmid, and a 70-fold enhancement was seen at the dose of $40 \mathrm{ng} /$ well. When NIH-3T3 cells were tested, luc expression increased in a dose-dependent manner, plateauing at $20 \mathrm{ng} /$ well, which was 30-fold over the control. AP-1 (equimolar c-Fos and c-Jun) plasmid also accelerated the luc expression by the CMV-TTT promoter (Fig. 3D). Again, HEK 293 cells showed a linear increase of luc activity corresponding to an increase of cotransfected AP-1. However, in NIH-3T3 cells, luc activity reached a peak that was $<30$-fold over the control. These observations suggested that doseresponse kinetics depends upon the nature of the cell lines.

To further characterize the action of the supercharging promoter system, similar experiments were repeated with various cell lines (Table II). It was again noteworthy that the system performance depended on the type of cell line, suggesting that i) compatibility of cis- and trans-acting factors for gene expression machinery of the individual cell type and ii) endogenous cofactor(s) determine the magnitude of resultant transgene expression. The combination of the CMV-KKK promoter and RelA showed a robust expression in $\mathrm{C} 6$ and a moderate effect in HeLa, HepG2, A431, and C2C12 cells, while that of the CMV-TTT promoter and Ap-1 demonstrated a good performance in ST-2, C6, and C2C12 cells. No enhancement was observed with any of these promoters in CHO-K1 cells. However, the substantial effect seen in $\mathrm{C} 2 \mathrm{C} 12$ myoblast inspired us to apply the strategy in vivo, since the muscle is a major target for in vivo gene transfer.

Transgene expression by the supercharging promoter system in vivo. We next examined whether the TF-supercharging promoter system can improve the magnitude and longevity of xenogeneic protein expression in mouse muscle. Human placental secretory alkaline phosphatase (huSEAP) that has immunogenicity in mice was employed as a reporter gene. SEAP activity was monitored by a plasma-based assay that allowed long-term surveillance of gene expression profile without sacrificing animals. It is an essential requirement for the system that reporter and TF plasmid is coexistent in individual cells. Two independent plasmids can be transferred into an individual cell by conventional transfection reagents in vitro, however, co-transfection with two plasmids into a single cell is unlikely in vivo. To pursue a maximal effect of the system in vivo, we constructed plasmids that carry both a 

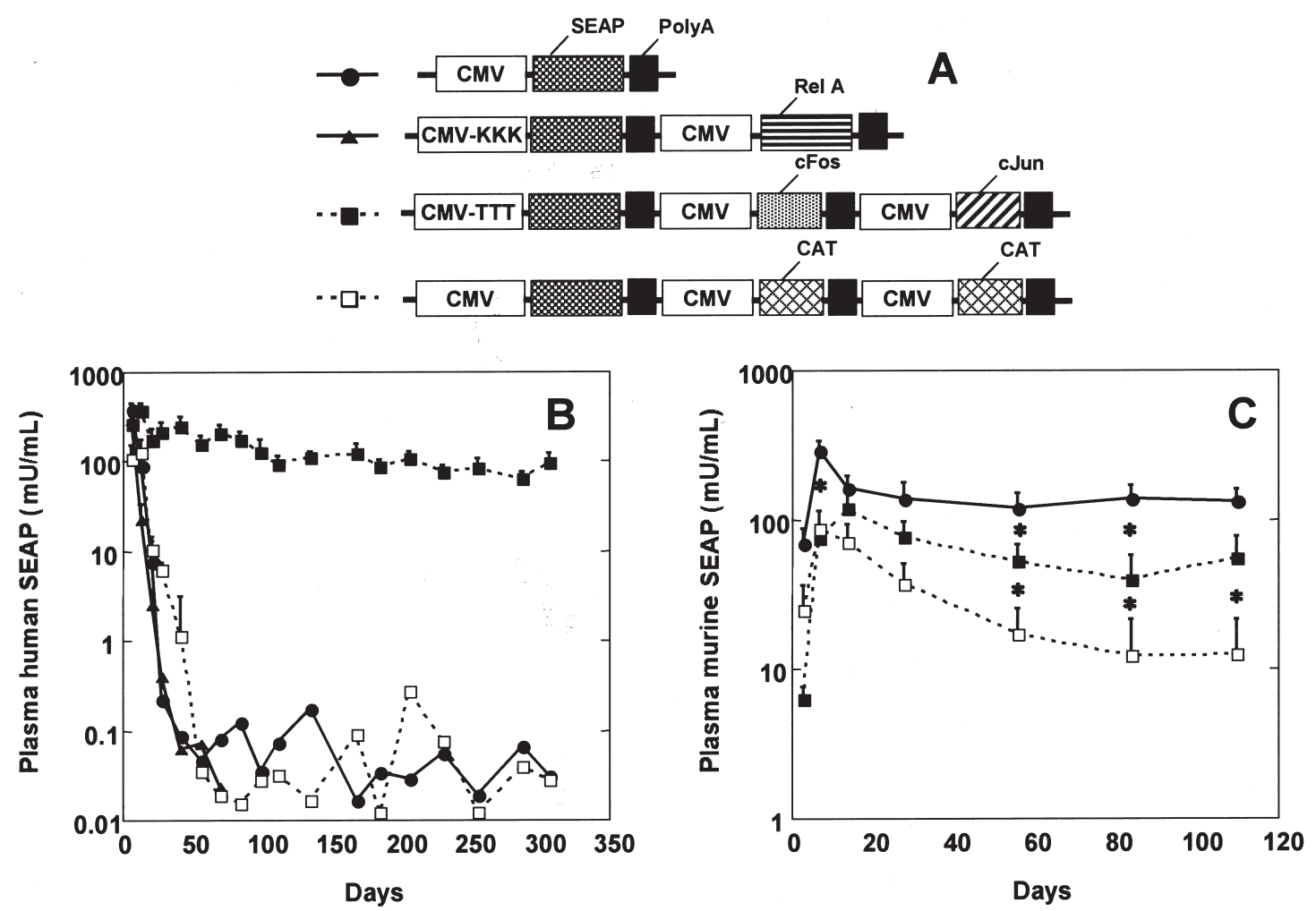

Figure 4. (A) Diagram of the plasmid vectors used in the in vivo experiment. CMV, cytomegalovirus immediate early gene enhancer/promoter plus intron A; SEAP, human or murine secretory alkaline phosphatase; polyA, bovine growth hormone polyadenylation signal; CAT, chloramphenicol acetyltransferase. Surveillance of plasma SEAP activity in BALB/c. The mice received the indicated plasmid expressing human (B) or murine (C) SEAP through electrotransfer. The mice ( 8 mice/group) were transfected with $50 \mu \mathrm{g}$ of each plasmid by intramuscular electrotransfer at day 0 , and plasma SEAP activity was monitored continuously. Plasma from eight mice in each group was assayed. The data shown are averages of triplicated measurements and the standard deviations. *Significant difference between two groups at the specific time point $(\mathrm{p}<0.05)$. An independent experiment showed a similar result.

reporter and TF gene in a single backbone by using a multiple promoter vector (21) (Fig. 4A). For AP-1, a triple promoter vector was constructed to express SEAP and c-Fos/c-Jun heterodimer simultaneously. Then quadriceps femoris muscle was transfected with this huSEAP expression plasmid by electroporation and SEAP activities were monitored consecutively for 10 months (Fig. 4B). The tricistronic vector that carries CMV-TTT promoter-driven SEAP and wild-type promoter-driven c-Fos and c-Jun sustained high-level SEAP expression throughout the observation period. Bicistronic vector that carried CMV-KKK/SEAP and CMV/RelA showed neither enhanced nor prolonged huSEAP expression (observation was truncated at day 56), exhibiting a striking difference of the system performance between in vitro and in vivo. A conventional huSEAP expression vector, in which the huSEAP gene is regulated by naïve CMV promoter, reached a peak close to the level obtained by the tricistronic vector, but plasma SEAP activity decreased rapidly and then sunk below the detection limit. The size-matched control for the tricistronic vector, which comprised of naïve CMV promoterdriven huSEAP and two copies of chloramphenicol acetyl transferase expression cassette, showed an expression profile that is similar to the conventional huSEAP expression vector. Thus, our novel approach is validated as useful in pursuing high and long-lasting expression of huSEAP in mice.

To clarify the relationship between performance of the AP-1-mediated supercharging promoter system and immunogenicity of a transgene, xenogeneic huSEAP was replaced with allogeneic muSEAP, and then SEAP expression profiles were monitored consecutively (Fig. 4C). A marked difference was observed regarding the longevity of SEAP expression depending upon the origin of SEAP. All three vectors used in the muSEAP experiment showed a sustained expression. It is noteworthy that the level of SEAP expression by a conventional plasmid was higher than that by the vector installed with AP-1mediated supercharging promoter throughout the observation period. However, the vector installed with AP-1-mediated supercharging promoter exhibited a higher level of SEAP expression than the tricistronic vector that has no supercharging promoter system, this difference might be explained by transactivation of CMV-TTT promoter by AP-1. The reason for the stronger muSEAP expression by the conventional promoter than by the supercharging promoter might be the plasmid size, as it is likely that the conventional muSEAP vector $(5.4 \mathrm{~kb})$ enters into a cell more efficiently than the AP-1-mediated supercharging promoter vector $(12 \mathrm{~kb})$. The above results suggest that the system may be suited for gene therapy targeting congenital protein deficiencies in which a patient develops an immune response against a therapeutic protein during enzyme replacement therapy.

Verification of immune-mediated clearance of huSEAP expression in vivo. To examine whether the clearance of huSEAP is attributed to an immune-mediated mechanism, huSEAP expression by a naïve CMV promoter was compared between immunocompetent and immunodeficient mice (Fig. 5). 


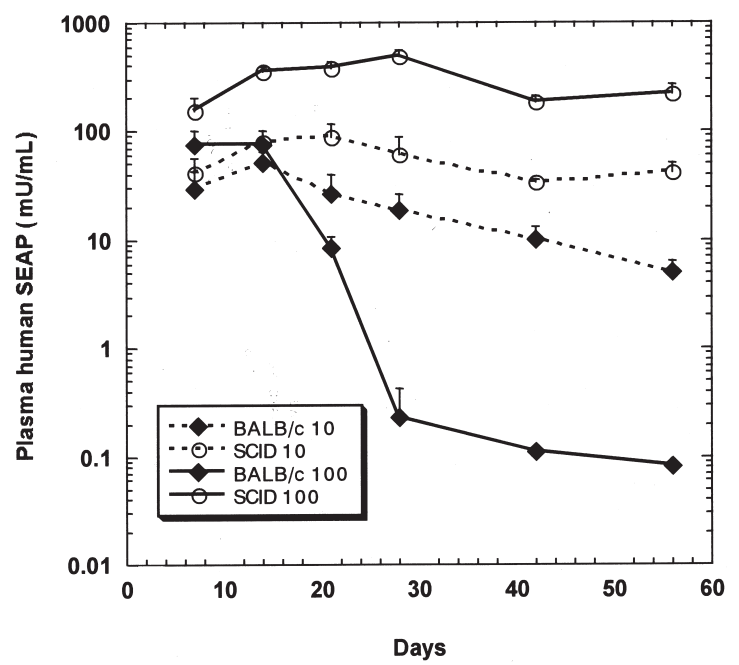

Figure 5. Human SEAP expression profile in the immunodeficient SCID (open circle) and immunocompetent BALB/c mice (closed circle). The mice ( 8 mice/group) were transfected with the indicated doses of $\mathrm{CMV} / \mathrm{huSEAP}$ plasmid by intramuscular electrotransfer at day 0 , and the plasma SEAP activity was monitored continuously. Plasma from eight mice in each group was pooled and assayed. The data shown are averages of triplicated measurements and standard deviations. An independent experiment showed a similar result.

As expected, in immunodeficient SCID mice, no loss of plasma SEAP activity was observed. Expression from $100 \mu \mathrm{g}$ of CMV/huSEAP was much higher than that from $10 \mu \mathrm{g}$ of the same plasmid, i.e. dose-dependent huSEAP activity is preserved. In contrast, in immunocompetent BALB/c mice, SEAP expression from $100 \mu \mathrm{g}$ of CMV/huSEAP was initially higher than that from $10 \mu \mathrm{g}$ of CMV/huSEAP, however, expression from $100 \mu \mathrm{g}$ of the vector had fallen dramatically to almost basal level within 28 days. This suggests that stronger transgene expression elicits a stronger immune response to a gene product, which interferes with later transgene expression. We therefore verified that huSEAP clearance occurred by an immune-mediated mechanism. Accordingly, it is proved that the AP-1-mediated supercharging promoter system maintains huSEAP expression against the immune-mediated clearance, further suggesting that the system fulfilled long-lasting gene expression not only by improving transcriptional activity but also by induction of immunological tolerance to huSEAP.

Comparison of mRNA expression level by conventional and AP-1-mediated supercharging promoter. To elucidate the mechanism underlying the induction of immunological tolerance to a xenogeneic gene achieved by AP-1-mediated supercharging promoter, transgene expression by conventional and supercharging promoter was analyzed in the lymph nodes and muscle at the messenger RNA level. In this experiment, non-immunogenic mouse SEAP is chosen as a reporter, since its expression is not subject to immune-mediated clearance and can reflect the net effect of transcriptional performance. As Table III shows, it is obvious that the standard CMV promoter can achieve higher mRNA expression than the supercharging promoter system not only in the lymph nodes but also in the muscles. At day 1, the supercharging promoter showed a significantly lower mRNA expression than the conventional promoter in the lymph nodes, which could be
Table III. Mouse SEAP mRNA expression in muscle and lymph nodes quantified by real-time RT-PCR.

\begin{tabular}{cccccc}
\hline & \multicolumn{2}{c}{ Naïve CMV promoter } & & \multicolumn{2}{c}{ CMV-TTT plus Ap-1 } \\
\cline { 2 - 3 } \cline { 5 - 6 } & Muscle & Lymph nodes & & Muscle & Lymph nodes \\
\hline Day 1 & $548.6 \pm 99.2^{\mathrm{a}}$ & $8.3 \pm 2.5^{\mathrm{a}}$ & & $64.1 \pm 14.3$ & $1.6 \pm 0.3$ \\
Day 3 & $445.3 \pm 73.6^{\mathrm{a}}$ & $1.0 \pm 0.3$ & & $20.9 \pm 12.1$ & $0.9 \pm 0.2$ \\
\hline
\end{tabular}

Mice were transfected with the plasmid expressing mouse SEAP under the indicated transcriptional control by electroporation, and the injected muscle and inguinal lymph nodes were harvested at day 1 and 3. Mouse SEAP mRNA was quantified by real-time PCR after RNA extraction and reverse transcription. The data shown are mean of five animals \pm standard errors. A repeated experiment

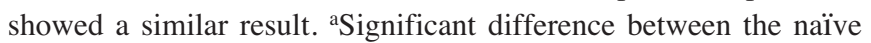
CMV and CMV-TTT group at the same time point.

responsible for the induction of immunological tolerance to a transgene. A markedly different transgene mRNA expression seen in the muscle might also play a role in determining whether a transgene product is recognized or passed over by the host immune system.

\section{Discussion}

The present report describes initial results from experiments in which our supercharging promoter system tested whether this strategy can ameliorate transgene expression in cell cultures and in mice. A major achievement in this study is the establishment of a strategy that is capable of sustaining high-level transgene expression following electroporationmediated plasmid transfer into the muscle. In the attempt to seek effective gene therapy using plasmid vectors, the muscle has been a major target because it is easily accessible and efficiently secretes the transgene product into the systemic circulation (24). Thus, amelioration of gene expression in the muscle might contribute to the progress of gene therapy targeting congenital disorders that can be treated with a therapeutic gene product in the circulation, i.e. hemophilia (25) and lysosomal storage diseases (26). In fact, previous studies have shown that intramuscular plasmid injection results in the production of therapeutic protein in hemophilia (3) and lysosomal storage diseases (6). However, the lack of persistent gene expression hampers efforts to supply therapeutic levels of target gene products. In this regard, our system might be suited for gene therapy targeting disorders that can be treated by supplying a deficient protein in the systemic circulation. Another concern in designing a gene therapy strategy targeting these congenital disorders is elicitation of immune responses to a deficient protein, as the patient's immune system might recognize a previously unexpressed protein as foreign. In fact, production of the antibody against deficient proteins has been reported in patients under enzyme replacement therapy for various congenital deficiencies such as hemophilia A and B (10), adenosine deaminase deficiency (11), mucopolysaccharidosis type I (12), Gaucher disease (13), Fabry disease (14), and Pompe disease (15). In these patients, antibodies may neutralize 
the bioactivity of the therapeutic protein, moreover, administration of the vector encoding therapeutic protein might augment an immune response to a transgene as seen in primeboost immunization of DNA vaccines. huSEAP is xenogeneic protein to mice and able to mimic a therapeutic gene product in a patient with congenital protein deficiency, as it has both bioactivity and immunogenicity in mice $(5,8)$. Hence, emphasis is given to the long-lasting expression of the huSEAP gene achieved by the TF-supercharging promoter consisting of CMV-TTT and AP-1, again providing favorable characteristics for gene therapy targeting congenital protein deficiencies, especially for patients who develop an immune response against a deficient protein. Following up the current study, the expression profile of therapeutic, but not reporter genes should be analysed using the CMV-TTT promoter and AP-1 to determine whether the system can exert its performance in a gene therapy model.

When allogeneic muSEAP was used as a reporter, SEAP expression persisted for more than 100 days regardless of the transcriptional control. This is in marked contrast to the rapid decrease of the transgene expression observed in huSEAPtreated animals. As noted, the strongest SEAP expression was attained by the conventional but not TF-supercharging promoter, presumably owing to the small plasmid size that is preferred for cellular uptake of plasmids. The AP-1-mediated supercharging promoter may therefore provide little help for improving transgene expression as long as a non-immunogenic gene is used. However, several studies of allogeneic factor VIII $(27-30)$, IX $(31,32)$, and erythropoietin $(33,34)$ gene transfer into animals indicate the risk of eliciting immune responses against a transgene, even if it originates from the same species. Thus we believe that the AP-1-mediated supercharging promoter system might confer considerable benefit for gene therapy targeting congenital protein deficiencies in which the therapeutic gene is recognized as foreign by the patient's immune system. The huSEAP expression profile observed in immunocompetent and immunodeficient mice displayed a striking contrast, decreasing huSEAP expression was seen only in immunocompetent mice. This finding indicates that huSEAP clearance is attributed to an immune-mediated mechanism, which is consistant with previous observations showing that plasma huSEAP level is in inverse proportion to anti-huSEAP antibody titre $(5,7,8)$. Quantitative real-time PCR analysis suggested that the avoidance of immune-activation by the AP-1-mediated supercharging promoter was achieved by lower mRNA expression shortly after DNA delivery in the regional lymph nodes where antigen presentation takes place. Physiologically, AP-1 regulates gene expression in response to a variety of physiological and pathological stimuli, including cytokines, growth factors, stress signals, infections, and chemical and physical stimuli (23). It is also a general understanding that the role of AP-1 depends upon the cell lineage, differentiation stage, and cellular microenvironment (35). Further study is necessary to clarify whether this approach causes potential adverse reactions such as mutagenesis and tumor.

The TF-supercharging promoter system that consists of CMV-KKK and RelA failed to ameliorate transgene expression in vivo while it demonstrated a good performance in vitro. The physiological role of RelA might account for the different performance seen in vitro and in vivo. Since RelA is a key transcription factor in initiating host defense against infection $(22,36)$, coexpression of RelA and SEAP in animals might activate the immune system and raise immune responses to SEAP. Previous studies have shown that myocytes expressing a foreign gene are subject to attack by inflammatory cells $(8,9)$, and transgene expression is mainly impaired by immunemediated mechanisms $(5,7,8)$. Thus, RelA is likely to facilitate immune-mediated elimination of cells bearing huSEAP.

There have been a few reports describing the strategies for transcriptional amplification using recombinant transcription factors (37-40). Among these approaches, the fusion protein of yeast GAL4 and the herpes simplex virus type 1 VP16 (GAL4-VP16) (41) is preferentially used (37-39), as it can elicit a robust transcriptional activity on a GAL4-responsive promoter. However, none of these studies showed sustained transgene expression by the GAL4-VP16-mediated transcription activation system. Therefore our findings represent the first demonstration that the recombinant TF-mediated transcriptional regulation system succeeded in increasing the longevity, but not magnitude of transgene expression. The present study may open a new avenue for manipulating the regulation of transgene expression. The strategy taken in this study might confer a considerable benefit for gene therapy targeting congenital protein deficiencies in which the therapeutic gene is recognized as foreign by the patient's immune system.

\section{References}

1. Boshart M, Weber F, Jahn G, Dorsch-Hasler K, Fleckenstein B and Schaffner W: A very strong enhancer is located upstream of an immediate early gene of human cytomegalovirus. Cell 41: 521-530, 1985 .

2. Miyazaki J, Takaki S, Araki K, Tashiro F, Tominaga A, Takatsu K and Yamamura K: Expression vector system based on the chicken beta-actin promoter directs efficient production of interleukin-5. Gene 79: 269-277, 1989.

3. Bettan M, Emmanuel F, Darteil R, Caillaud JM, Soubrier F, Delaere P, Branelec D, Mahfoudi A, Duverger N and Scherman D: High-level protein secretion into blood circulation after electric pulse-mediated gene transfer into skeletal muscle. Mol Ther 2: 204-210, 2000.

4. Bettan M, Darteil R and Scherman D: Secreted human placental alkaline phosphatase as a reporter gene for in vivo gene transfer. Anal Biochem 271: 187-189, 1999.

5. Chastain M, Simon AJ, Soper KA, Holder DJ, Montgomery DL, Sagar SL, Casimiro DR and Middaugh CR: Antigen levels and antibody titers after DNA vaccination. J Pharm Sci 90: 474-484, 2001.

6. Yew NS, Przybylska M, Ziegler RJ, Liu D and Cheng SH: High and sustained transgene expression in vivo from plasmid vectors containing a hybrid ubiquitin promoter. Mol Ther 4: 75-82, 2001. (Erratum in Mol Ther 4: 280, 2001).

7. Maelandsmo GM, Ross PJ, Pavliv M, Meulenbroek RA, Evelegh C, Muruve DA, Graham FL and Parks RJ: Use of a murine secreted alkaline phosphatase as a non-immunogenic reporter gene in mice. J Gene Med 7: 307-315, 2005.

8. Gronevik E, von Steyern FV, Kalhovde JM, Tjelle TE and Mathiesen I: Gene expression and immune response kinetics using electroporation-mediated DNA delivery to muscle. J Gene Med 7: 218-227, 2005.

9. Payette PJ, Weeratna RD, McCluskie MJ and Davis HL: Immunemediated destruction of transfected myocytes following DNA vaccination occurs via multiple mechanisms. Gene Ther 8: 1395-1400, 2001

10. Scharrer I, Bray GL and Neutzling O: Incidence of inhibitors in haemophilia A patients - a review of recent studies of recombinant and plasma-derived factor VIII concentrates. Haemophilia 5: 145-154, 1999. 
11. Chaffee S, Mary A, Stiehm ER, Girault D, Fischer A and Hershfield MS: IgG antibody response to polyethylene glycolmodified adenosine deaminase in patients with adenosine deaminase deficiency. J Clin Invest 89: 1643-1651, 1992.

12. Kakkis ED, Muenzer J, Tiller GE, Waber L, Belmont J, Passage M, Izykowski B, Phillips J, Doroshow R, Walot I, Hoft R and Neufeld EF: Enzyme-replacement therapy in mucopolysaccharidosis I. New England J Med 344: 182-188, 2001.

13. Richards SM, Olson TA and McPherson JM: Antibody response in patients with Gaucher disease after repeated infusion with macrophage-targeted glucocerebrosidase. Blood 82: 1402-1409, 1993.

14. Eng CM, Guffon N, Wilcox WR, Germain DP, Lee P, Waldek S, Caplan L, Linthorst GE, Desnick RJ and International Collaborative Fabry Disease Study Group: Safety and efficacy of recombinant human alpha-galactosidase A - replacement therapy in Fabry's disease. New England J Med 345: 9-16, 2001.

15. Amalfitano A, Bengur AR, Morse RP, Majure JM, Case LE, Veerling DL, Mackey J, Kishnani P, Smith W, McVie-Wylie A, Sullivan JA, Hoganson GE, Phillips JA III, Schaefer GB, Charrow J, Ware RE, Bossen EH and Chen YT: Recombinant human acid alpha-glucosidase enzyme therapy for infantile glycogen storage disease type II: results of a phase I/II clinical trial. Genet Med 3: 132-138, 2001.

16. Saukkonen $\mathrm{K}$ and Hemminki A: Tissue-specific promoters for cancer gene therapy. Expert Opin Biol Ther 4: 683-696, 2004.

17. Papadakis ED, Nicklin SA, Baker AH and White SJ: Promoters and control elements: designing expression cassettes for gene therapy. Current Gene Ther 4: 89-113, 2004

18. Ross TM, Xu Y, Bright RA and Robinson HL: C3d enhancement of antibodies to hemagglutinin accelerates protection against influenza virus challenge. Nat Immunol 1: 127-131, 2000.

19. Wang M, Orsini C, Casanova D, Millan JL, Mahfoudi A and Thuillier V: MUSEAP, a novel reporter gene for the study of long-term gene expression in immunocompetent mice. Gene 279: 99-108, 2001.

20. Takeshita F, Suzuki K, Sasaki S, Ishii N, Klinman DM and Ishii KJ: Transcriptional regulation of the human TLR9 gene. J Immunol 173: 2552-2561, 2004.

21. Sasaki S, Amara RR, Oran AE, Smith JM and Robinson HL: Apoptosis-mediated enhancement of DNA-raised immune responses by mutant caspases. Nat Biotechnol 19: 543-547, 2001.

22. Li Q and Verma IM: NF-kappaB regulation in the immune system. Nature Rev Immunol 2: 725-734, 2002.

23. Eferl R and Wagner EF: AP-1: a double-edged sword in tumorigenesis. Nat Rev Cancer 3: 859-868, 2003.

24. Herweijer $\mathrm{H}$ and Wolff JA: Progress and prospects: naked DNA gene transfer and therapy. Gene Ther 10: 453-458, 2003.

25. Walsh CE: Gene therapy progress and prospects: gene therapy for the hemophilias. Gene Ther 10: 999-1003, 2003.

26. Cheng SH and Smith AE: Gene therapy progress and prospects: gene therapy of lysosomal storage disorders. Gene Ther 10 1275-1281, 2003
27. Qian J, Collins M, Sharpe AH and Hoyer LW: Prevention and treatment of factor VIII inhibitors in murine hemophilia A. Blood 95: 1324-1329, 2000.

28. Wu H, Reding M, Qian J, Okita DK, Parker E, Lollar P, Hoyer LW and Conti-Fine BM: Mechanism of the immune response to human factor VIII in murine hemophilia A. Thromb Haemost 85: 125-133, 2001.

29. Sarkar R, Gao GP, Chirmule N, Tazelaar J and Kazazian HH Jr: Partial correction of murine hemophilia A with neo-antigenic murine factor VIII. Hum Gene Ther 11: 881-894, 2000.

30. Ye P, Thompson AR, Sarkar R, Shen Z, Lillicrap DP, Kaufman RJ, Ochs HD, Rawlings DJ and Miao CH: Naked DNA transfer of Factor VIII induced transgene-specific, speciesindependent immune response in hemophilia A mice. Mol Ther 10: 117-126, 2004.

31. Fields PA, Kowalczyk DW, Arruda VR, Armstrong E, McCleland ML, Hagstrom JN, Pasi KJ, Ertl HC, Herzog RW and High KA: Role of vector in activation of $\mathrm{T}$ cell subsets in immune responses against the secreted transgene product factor IX. Mol Ther 1: 225-235, 2000.

32. Herzog RW, Fields PA, Arruda VR, Brubaker JO, Armstrong E, McClintock D, Bellinger DA, Couto LB, Nichols TC and High KA: Influence of vector dose on factor IX-specific T and $\mathrm{B}$ cell responses in muscle-directed gene therapy. Hum Gene Ther 13: 1281-1291, 2002.

33. Chenuaud P, Larcher T, Rabinowitz JE, Provost N, Cherel Y, Casadevall N, Samulski RJ and Moullier P: Autoimmune anemia in macaques following erythropoietin gene therapy. Blood 103: 3303-3304, 2004.

34. Gao G, Lebherz C, Weiner DJ, Grant R, Calcedo R, McCullough B, Bagg A, Zhang Y and Wilson JM: Erythropoietin gene therapy leads to autoimmune anemia in macaques. Blood 103: 3300-3302, 2004.

35. Hess J, Angel P and Schorpp-Kistner M: AP-1 subunits: quarrel and harmony among siblings. J Cell Sci 117: 5965-5973, 2004.

36. Taniguchi T, Ogasawara K, Takaoka A and Tanaka N: IRF family of transcription factors as regulators of host defense. Ann Rev Immunol 19: 623-655, 2001.

37. Iyer M, Wu L, Carey M, Wang Y, Smallwood A and Gambhir SS Two-step transcriptional amplification as a method for imaging reporter gene expression using weak promoters. Proc Natl Acad Sci USA 98: 14595-14600, 2001.

38. Ray S, Paulmurugan R, Hildebrandt I, Iyer M, Wu L, Carey M, and Gambhir SS: Novel bidirectional vector strategy for amplification of therapeutic and reporter gene expression. Hum Gene Ther 15: 681-690, 2004

39. Nettelbeck DM, Jerome V and Muller R: A strategy for enhancing the transcriptional activity of weak cell typespecific promoters. Gene Ther 5: 1656-1664, 1998

40. Veelken H, Leutgeb B, Kulmburg P, Fiebig H-H, Mackensen A and Lindemann A: Enhancement of a constitutively active promoter for gene therapy by a positive feed-back transcriptional activator mechanism. Int J Mol Med 2: 423-428, 1998.

41. Sadowski I, Ma J, Triezenberg S and Ptashne M: GAL4-VP16 is an unusually potent transcriptional activator. Nature 335: 563-564, 1988. 